\title{
Molecularly Imprinted Electrochemical Sensor based on Bioinspired Au Microflowers for ultra-trace Cholesterol assay
}

\author{
Hua Yang ${ }^{\mathrm{a}}, \mathrm{Li} \mathrm{Li}^{\mathrm{a}}$, Yaping Ding ${ }^{1}$, a, c, Daixin Ye ${ }^{\mathrm{b}}$, Yingzi Wang ${ }^{\mathrm{a}}$, Shiqiang Cui ${ }^{\mathrm{a}}$, Lanfeng Liao ${ }^{\mathrm{a}}$

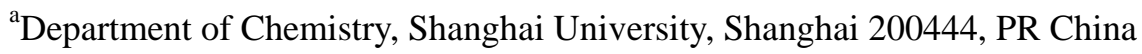 \\ ${ }^{b}$ Department of Chemistry and Molecular Biology, University of Gothenburg, Gothenburg S-41296, Sweden \\ 'Shanghai Key Laboratory of High Temperature Superconductors, Shanghai University, Shanghai 200444, PR China
}

\begin{abstract}
A novel imprinted sensor for ultra-trace cholesterol (CHO) detection based on electropolymerized aminothiophenol (ATP) molecularly imprinted polymer (MIP) on a glassy carbon electrode (GCE) modified with dopamine@graphene (DGr) and bioinspired Au microflowers has been developed in this work. As the specific recognition element, the bioinspired Au microflowers were formed by Au nanoparticles (AuNPs) and wrapped by bionic polydopamine film (PDA) through electropolymerization method. These excellent biocompatible materials could capture the target $\mathrm{CHO}$ effectively. The morphology of the MIP modified electrode was characterized by scanning electron microscopy (SEM) and atomic force microscope (AFM). The hydrogen-bonding interaction between templates and monomers was characterized by ultraviolet spectroscopy. Under the optimal experimental conditions, the sensor's linear response range was between $10^{-18}$ and $10^{-13} \mathrm{M}$, with a detection limit of $3.3 \times 10^{-19} \mathrm{M}$, which was much more sensitive than most available $\mathrm{CHO}$ detection methods in previously reports. Moreover, the MIP sensor exhibited high sensitivity for $\mathrm{CHO}$, low interference, and good stability. The human serum samples analysis confirmed the applicability of this MIP sensor to quantitative analysis of ultra-trace CHO.
\end{abstract}

Keywords: Molecularly imprinted electrochemical sensor; Polydopamine; Au microflowers; Cholesterol

\footnotetext{
* Corresponding author: Tel: +86-21-66134734; Fax: +86-21-66132797

Address: Department of Chemistry, Shanghai University, Shanghai 200444, PR China

E-mail address: wdingyp@sina.com
} 


\section{Introduction}

Coronary artery disease is the number one cause of death in all developed countries. Several studies have demonstrated the increased risk of cardiovascular diseases including arteriosclerosis is strongly correlated with high cholesterol (CHO) levels (Ahmadraji and Killard, 2013). Thus, the determination of $\mathrm{CHO}$ levels in food and blood has been increasingly important for clinical analysis/diagnosis. Conventionally, $\mathrm{CHO}$ has been detected by various methods, such as HPLC (Dong et al., 2012), gas chromatography (García-Llatas et al., 2012), CHO biosensors (Tripathy et al., 2014) and electrophoretic chips (Ruecha et al., 2011). While these methods require complex procedures for precipitation of lipoprotein fractions and suffer from low specificity, instability of reagents and high cost. Thus, it is great urgency to develop a method with high sensitivity and selectivity, good stability and low cost for estimation of $\mathrm{CHO}$.

Molecularly imprinted electrochemical sensors (MIECS), which replace biological receptors with wholly synthetic analogues as a recognition element in chemo/biosensors, are expected as less expensive apparatus and direct analytical techniques for high sensitivity, good selectivity and rapid estimation of different molecules ranging from small hazardous molecules to biomacromolecules (Riskin et al., 2008; Altintas et al., 2015; Malitesta et al., 2012). However, conventional synthetic methods may be limiting in terms of mass transfer, incomplete template removal and rebinding kinetics in sensor applications. In order to overcome this problem, electrochemical polymerization has been introduced due to its advantages that the thickness of the polymer can be controlled by various parameters such as current density and applied voltage, resulting in a uniform and compact coating of the polymer on the electrode surface. An increase number of research papers in the field of MIECS indicate that improvement in the effective protocols for molecularly imprinted polymer s'(MIP) immobilization on the electrode surface and high affinity for the target analyte is necessary 
(Suryanarayanan et al., 2010). Ji et al. (2015) prepared MIP films on a glassy carbon electrode modified with multi-walled carbon nanotubes and $\mathrm{Au}$ nanoparticles (AuNPs). They combined surface molecular self-assembly with copoly- merization of poly-aminothiophenol, CHO and AuNPs to maximize the amount of effective imprinted sites and showed a linear range between $10^{-13} \mathrm{M}$ and $10^{-9} \mathrm{M}$. Herein, we developed a novel bioinspired MIECS with good biocompatibility to capture biomacromolecules and could get superb sensitivity. Moreover, bioinspired polydopamine (PDA) films are used to achieve better imprinted effect and waste less time in preparation than self-assembly monolayers.

Polydopamine (PDA) has been implemented as an excellent surface-adherent material for multifunctional coatings of membrane surfaces. Bio-inspired research has found that dopamine (DA) (3,4-dihydroxy phenethylamine) can self-polymerize in marine condition (i.e. alkaline solution) and form not only strong adhesion but biocompatible layer on the substrate on which it is grown (Lee et al., 2007). Therefore, PDA could immobilize graphene (Gr) onto the modified electrode. Furthermore, the PDA layer has catechol groups and amino groups. Under oxidizing conditions, catechols react with thiols and amines via Michael addition or Schiff base reactions, aminos can react with hydroxyls and caoboxyls (LaVoie et al., 2005). Thus, the PDA layer could interact with electropolymerized ATP molecular imprinting films through intermolecular forces between ATP and PDA. PDA film has a cross-linked structure, on which could generate stable three-dimensional imprinting sites and capture more template $\mathrm{CHO}$ molecules. What's more, the metal binding ability of catechols supports metallization in situ (Pierpont et al., 1994), which could stabilize AuNPs. Therefore, the resultant PDA coating films can be regarded as a versatile platform for secondary surface modified reactions, prompting to nanoparticles stabilization, surface functionalization, 
self-assembled monolayer, and bioactive molecules immobilization (Liu et al., 2013; Zhang et al., 2014; Cao et al., 2015; Zan et al., 2016). Until now there are only a few researches on PDA based MIP, however, most of which concerned DA as functional monomers in MIP. Zhou et al. (2010) demonstrated a facile approach for imprinting protein based on self-polymerization of DA as functional monomers in the presence of template protein on the $\mathrm{Fe}_{3} \mathrm{O}_{4}$ NPs. Liu et al. (2013) prepared a novel electrosynthesized PDA-imprinted film as a recognition element for the capacitive sensing of nicotine. However, $\mathrm{CHO}$ is a hydrophobic organic molecule and could not dissolved in aqueous solution which DA could dissolve in. It is impossible for DA to act as functional monomers and react with CHO fully through non-covalent interactions in aqueous solution. In this work, we applied the electrosynthesized PDA as a bioinspired multifunctional modified materials in MIP for capture CHO for the first time.

It is well-known that the sensitivity of the imprinted sensor is dictated by the amount of effective imprinted sites on the sensor surface. To further increase the amount of effective imprinted sites, functional materials are combined with MIECS to achieve a synergistic effect. AuNPs are chosen due to their large specific surface area, good biocompatibility, and high conductivity (Xu et al., 2004). Moreover, AuNPs could act as crosslinkers to form polymeric networks by Au - S in MIP preparation. Xie et al. (2010) reported electropolymerized polyaminothiophenol molecular imprinting membranes at the surface of gold nanoparticles modified glassy carbon electrode by the formation of Au - S bonds to detect pesticide chlorpyrifos. The larger surface area of a AuNPs modified electrode produced a high ratio of imprinted sites and, thus, provides an ultrasensitive electrochemical detection of organophosphate pesticide (Xie et al., 2010). Gr is an excellent candidate as supported material for preparing molecularly imprinted materials because of their excellent conductivity, high 
surface area and ease of modification.

In this work, considering the above-mentioned attractive properties of Gr, AuNPs, and PDA, a novel AuNPs-PDA-DGr/GCE modified ATP MIP electrode for ultra-trace CHO determination was prepared by using DA@Gr (DGr) and bioinspired Au microflowers as substrate material. In this preparation procedure, DA was used not only to improve the dispersibility of Gr but also to produce a versatile platform on Gr surface for prompting to stabilize AuNPs and MIP membranes. AuNPs, wrapped by bioinspired PDA film, grew to versatile $\mathrm{Au}$ microflowers and produced a high ratio of imprinted sites, which could improve the sensitivity of the MIP sensor. Furthermore, versatile Au microflowers can immobilize MIPs film through intermolecular forces between ATP and PDA, as well as $\mathrm{Au}-\mathrm{S}$ between AuNPs and ATP. The influential parameters affecting the adsorption of $\mathrm{CHO}$ such as $\mathrm{pH}$ of electropolymerized DA solution, elution conditions, adsorption time, temperature, scan cycles and scan rates were optimized. In the optimum condition, this electrochemical sensor showed good reproducibility, high stability and wide linear range, which can be potentially exploited for the detection of trace $\mathrm{CHO}$ in human serum.

\section{Experimental}

\subsection{Apparatus}

UV-vis spectroscopy were recorded on UV-2501PC system (Shimadzu Corporation). Fourier Transform Infrared Spectrometer (FT-IR) were carried out using AVATAR370 FT-IR system (Thermo Nicolet Corporation). Scanning electron microscopy (SEM) images were carried out using a scanning electron microscope (JSM-6700F, $15.0 \mathrm{kV}$ ). Atomic force microscopy (AFM) images were taken in tapping mode with the Dimension ${ }^{\circledR} \mathrm{Edge}^{\mathrm{TM}}$.

Electrochemical measurements were performed using a CHI660d workstation (Chenhua 
Corpration, Shanghai, China) connected to a personal computer. A conventional three-electrode system consisting of a bare or modified GCE ( $3 \mathrm{~mm}$ in diameter) as a working electrode, a saturated calomel electrode as the reference electrode, and a platinum electrode as the auxiliary electrode was employed in a solution containing $5 \times 10^{-3} \mathrm{~mol} \mathrm{~L}^{-1} \mathrm{Fe}(\mathrm{CN})_{6}{ }^{3-/ 4-}$ consisting of $\mathrm{K}_{3} \mathrm{Fe}(\mathrm{CN})_{6}, \mathrm{~K}_{4} \mathrm{Fe}(\mathrm{CN})_{6}$, and $0.1 \mathrm{M} \mathrm{KCl}$.

\subsection{Reagents}

Gr was purchased from XFNANO Materials Tech Co., Ltd (Nanjing, China). CHO, tetrachloroaurate (III) acid $\left(\mathrm{HAuCl}_{4}\right)$ was purchased from Sinopharm Chemical Reagent Co., Ltd. p-ATP, DA and $\mathrm{LiClO}_{4}$ were purchased from Aladdin Chemical Reagent Co., Ltd. (Shanghai, China). All other reagents were commercially available as analytical reagent grade and were used without further treatment. Standard solutions of $\mathrm{CHO}$ were prepared in ethanol. Solutions of $1 \mathrm{mg} / \mathrm{ml} \mathrm{Gr}$ and DGr were prepared in $\mathrm{N}, \mathrm{N}$ - dimethylformamide (DMF). Phosphate buffer solution (PBS) was prepared from $\mathrm{KH}_{2} \mathrm{PO}_{4}$ and $\mathrm{K}_{2} \mathrm{HPO}_{4}$ (Sinopharm Chemical Reagent Co., Ltd.) with doubly distilled water. $\mathrm{LiClO}_{4}(0.05 \mathrm{M})$ was used as the supporting electrolyte.

\subsection{Fabrication of MIP-AuNPs-PDA-DGr/GCE}

The preparation of DGr and fabrication of AuNPs-PDA-DGr modified GCE were detailed in supplementary material.

The AuNPs-PDA-DGr/GCE was immersed in the ethanol electrolyte solution containing $10 \mathrm{mM}$ ATP, $10 \mathrm{mM} \mathrm{CHO}$ and $0.05 \mathrm{M} \mathrm{LiClO}_{4}$. The electropolymerization was performed by seven consecutive cyclic scans in the potential range of -0.2 and $+0.6 \mathrm{~V}$ at a scan rate of $75 \mathrm{mV} \mathrm{s}{ }^{-1}$. After electropolymerization, the modified GCE was immersed in an ethanol solution containing 0.5 mol $\mathrm{L}^{-1} \mathrm{HCl}$ and treated at $-0.1 \mathrm{~V}$ constant potential for $40 \mathrm{~s}$ to remove the $\mathrm{CHO}$ template molecules. 
Then the MIP-AuNPs-PDA-DGr/GCE electrode was rinsed with doubly distilled water and ethanol and dried under nitrogen for further use. As a control, the nonimprinted electrode followed the same procedure but without a template molecules.

\subsection{Electrochemical measurements}

The modified electrode was dipped into a solution of 5 mmol L ${ }^{-1}\left[\mathrm{Fe}(\mathrm{CN})_{6}\right]^{3-/ 4-}$ with $0.1 \mathrm{~mol} \mathrm{~L}^{-1}$ $\mathrm{KCl}$ as the supporting electrolyte. $\mathrm{CV}$ and DPV measurements was recorded between -0.2 to $+0.6 \mathrm{~V}$. $\mathrm{CV}$ of electropolymerization of the MIP film were performed between -0.2 and $+1.2 \mathrm{~V}$ at a scan rate of $75 \mathrm{mV} \mathrm{s}^{-1}$. The electrochemical impedance spectroscopy was scanned in $5 \mathrm{mM} \mathrm{Fe}(\mathrm{CN}){ }_{6}^{3-/ 4-}$ and $0.1 \mathrm{M} \mathrm{KCl}$ solution at the open circuit potential with a frequency range of $1-10^{5} \mathrm{~Hz}$ and an amplitude of $5 \mathrm{mV}$.

\section{Results and discussion}

\subsection{Preparatrion and Characterization of MIP-AuNPs-PDA-DGr/GCE}

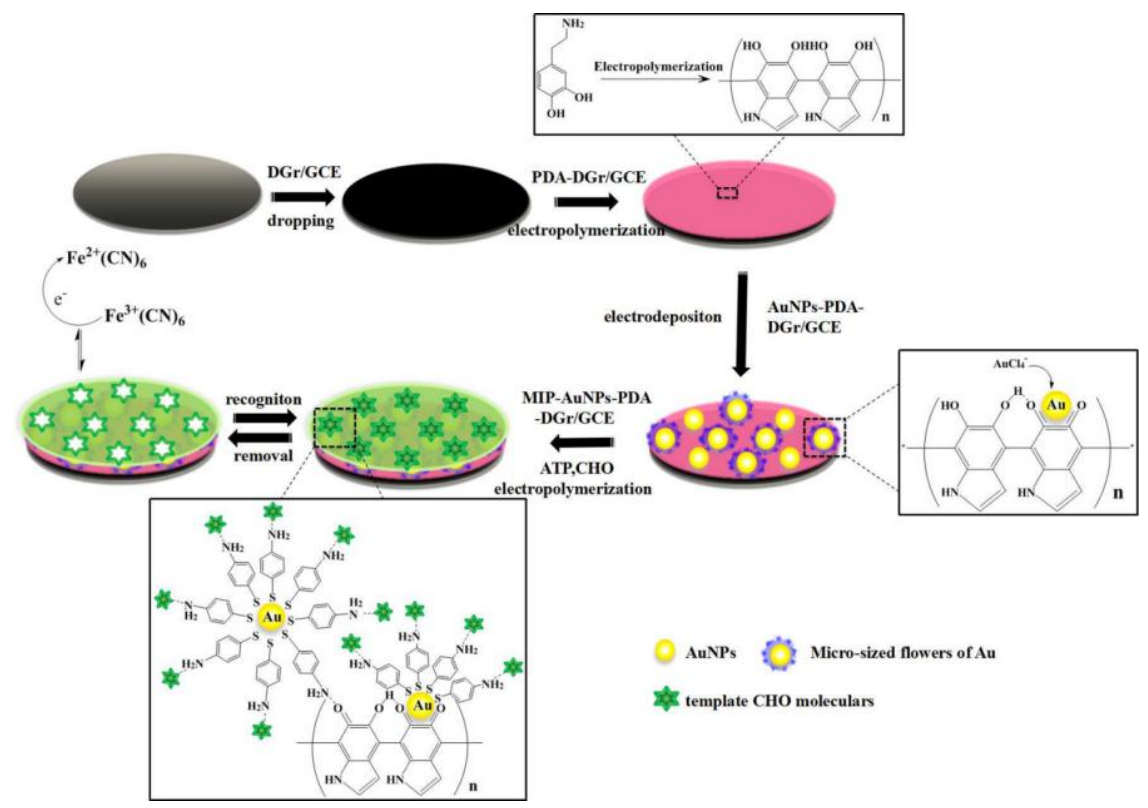

Scheme 1 The preparation process of MIP-AuNPs-PDA-DGr/GCE.

The fabrication procedure for MIP-AuNPs-PDA-DGr/GCE is illustrated in Scheme 1. The process can be summarized as four steps: (1)DGr suspension was dropped on the surface of GCE; 
(2)electropolymerization of DA and electrodeposition of AuNPs on the surface of DGr/GCE; (3)electropolymerization of ATP and CHO on the surface of AuNPs-PDA-DGr/GCE; (4)removal of the template $\mathrm{CHO}$ molecules from the MIP electrode. Firstly, Gr was mixed with DA in weak alkaline PBS (pH 8.5) to produce DGr. GCE was coated with $5 \mu \mathrm{L}$ of the resulting DGr suspension. In this procedure, DA acted as a reducing agent for Gr accompanied by the spontaneous depositon of a thin adherent PDA film. The dispersion and stability of DGr after modified had been enhanced compared to Gr. The SEM image of Gr and DGr are shown in Fig.1. As shown in Fig.1A and 1B, DGr (1B) was wrapped by PDA film, which had lighter colour than the Gr (1A). The purified DGr was characterized by FTIR. (the FTIR figure of Gr and DGr are shown in Fig.S1 in the supporting information)

Secondly, the DGr/GCE was modified with PDA and AuNPs. The SEM image of the AuNPs-PDA-DGr/GCE is shown in Fig.1C. As shown in Fig.1C, AuNPs wrapped with PDA film homogeneously distributed on the surface of the DGr and grew to Au microflowers, indicating that PDA effectively prevent the AuNPs from aggregation. The PDA layer has a great deal of catechol groups and amino groups, which can react with many chemical groups (such as thiols and aminos of the next MIP layer, the intermolecular interaction between ATP and PDA was confirmed by UV absorbance spectra and the figure is shown in Fig.S2 in the supporting information) and induce metal nanoparticles in situ reduction. $\mathrm{AuCl}_{4}{ }^{-}$ions in the solution of $\mathrm{HAuCl}_{4}$ are absorbed onto the PDA layer. Then $\mathrm{AuCl}_{4}{ }^{-}$ions are reduced to AuNPs by the catechol groups of PDA and constant potential method. Moreover, AuNPs are partially wrapped by PDA film and grow to Au microflowers. These AuNPs can interact with ATP through Au-S bonds between AuNPs and thiol groups of ATP. Moreover, these AuNPs also can enhance the electrochemical signal because they can plant many 
recognition sites on the modified electrode surface due to their large specific surface area. In addition, PDA can form strong adhesion layer on the surface of DGr to prevent shedding.

Finally, the MIP film was electropolymerized onto the modified electrode surface in the ethanol electrolyte solution containing $10 \mathrm{mM}$ ATP, $10 \mathrm{mM} \mathrm{CHO}$ and $0.05 \mathrm{M} \mathrm{LiClO}_{4}$ by seven consecutive cyclic scans in the potential range of -0.2 and $+0.6 \mathrm{~V}$ at a scan rate of $75 \mathrm{mV} \mathrm{s}^{-1}$. The $\mathrm{CV}$ of electropolymerization of MIP films is shown in the Fig.1E. As we can see, two irreversible oxidation peaks which are probably the electropolymerization of p-ATP at potentials of approximately $0.47 \mathrm{~V}$ and $0.95 \mathrm{~V}$ appeared during the first cycle and decreased gradually until they disappeared. The results indicate that the MIP membranes formed on the surface of the modified electrode. The decrease of the peaks may be related to the continual formation of electrochemical inactive MIP films which suppress the voltammetric response. We used ATP as monomer to form MIP with CHO in this work, which is because ATP can react with not only PDA and AuNPs through intermolecular forces and $\mathrm{Au}-\mathrm{S}$ bonds, but also $\mathrm{CHO}$ through hydrogen bond interactions between ATP and CHO. The interaction between ATP and CHO can be confirmed by UV absorbance spectra (Fig.1F). With the addition of $\mathrm{CHO}$, the maximum absorption peak of ATP was largely increased and the maximum absorption wavelength slightly shifted to blue wavelength. This result suggests the formation of hydrogen bonds between the amino groups of ATP and hydroxy groups of CHO. The SEM image of the MIP modified electrode surface is shown in the Figure 1D. The much rougher surface of the MIP modified electrode confirms the formation of MIP membranes and provides a large surface area for the absorption of template $\mathrm{CHO}$ molecules. The AFM images of the MIP/NIP surfaces are shown in Fig.S3. We can see a very rough surface morphology both for MIP and NIP. The formation of imprinted cavities cannot directly be detected by AFM, but the phase angle images of MIP and NIP 
can indicate a stiff and more compact NIP layer.
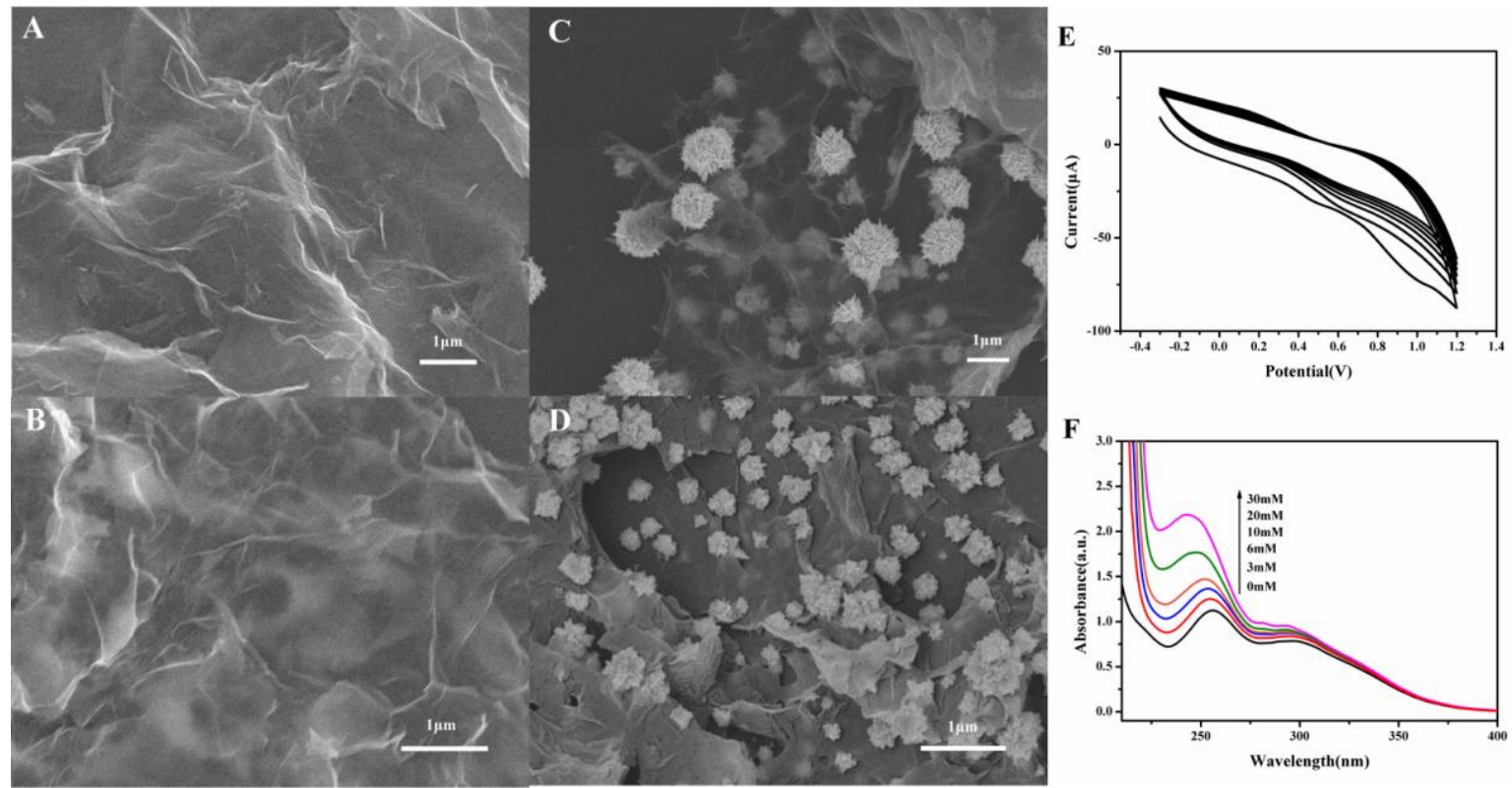

Fig.1 SEM image of (A) Gr/GCE, (B) DGr/GCE, (C) AuNPs-PDA-DGr/GCE, and (D) MIP-AuNPs-PDA-DGr /GCE. (E) Cyclic voltammograms for the co-polymerization of $10 \mathrm{mM}$ ATP and $10 \mathrm{mM} \mathrm{CHO}$ at AuNPs-PDA-DGr /GCE surface in ethanol solution containing $0.05 \mathrm{M} \mathrm{LiClO}_{4}$ : scan rate, $75 \mathrm{mV} \mathrm{s}^{-1}$; sweep cycle, 7. (F) UV spectra with increasing different amounts of CHO into $0.1 \mathrm{mM}$ ATP solution.

Fig.2A shows the $\mathrm{CV}$ of bare electrode and modified electrode in $\left.5 \mathrm{mmol} \mathrm{L}-1 \mathrm{Fe}(\mathrm{CN})_{6}\right]^{3-/ 4-}$ and $0.1 \mathrm{mmol} \mathrm{L}{ }^{-1} \mathrm{KCl}$. We can see that the bare electrode showed a quasi-reversible redox reaction. When the electrode modified with DGr, the redox peak currents increased. This result may be because of the excellent conductivity of DGr. The redox peak currents of PDA-DGr/GCE decreased may be contributed to the formation of PDA blocking the charge transfer of the redox probe. After AuNPs depositon onto the modified electrode, the redox peak currents increased may be due to a small increase of surface roughness and effective surface area. Moreover, the Fig.S4 shows the CV of the MIP/NIP modified electrode. Also $\left[\mathrm{Fe}(\mathrm{CN})_{6}\right]^{3-/ 4-}$ was used as a redox probe. The NIP modified electrode shows almost no redox peaks compared to the AuNPs-PDA-DGr/GCE. This is likely because the NIP films blocked the electron transfer. However, the MIP modified electrode shows a pair of redox peaks. This suggests that cavities were produced in the MIP films after the removal of template $\mathrm{CHO}$ molecules. These cavities made it possible for $\left[\mathrm{Fe}(\mathrm{CN})_{6}\right]^{3-/ 4-}$ to diffuse through the 
MIP films and to take place redox reaction on the modified electrode. Fig.2B shows the DPV of modified electrode. Also compared to the current response of AuNPs-PDA-DGr/GCE, the NIP modified electrode shows almost no redox peaks, indicating the formation of p-ATP films on the modified electrode. The peak currents of MIP modified electrode show a decrease in $\Delta \mathrm{Ip}(40 \mu \mathrm{A})$ compared to AuNPs-PDA-DGr/GCE and a increase in $\Delta \mathrm{Ip}(100 \mu \mathrm{A})$ compared to NIP modified electrode. These results confirm after template $\mathrm{CHO}$ molecules removing from the MIP films the cavities produced on the MIP films successfully.
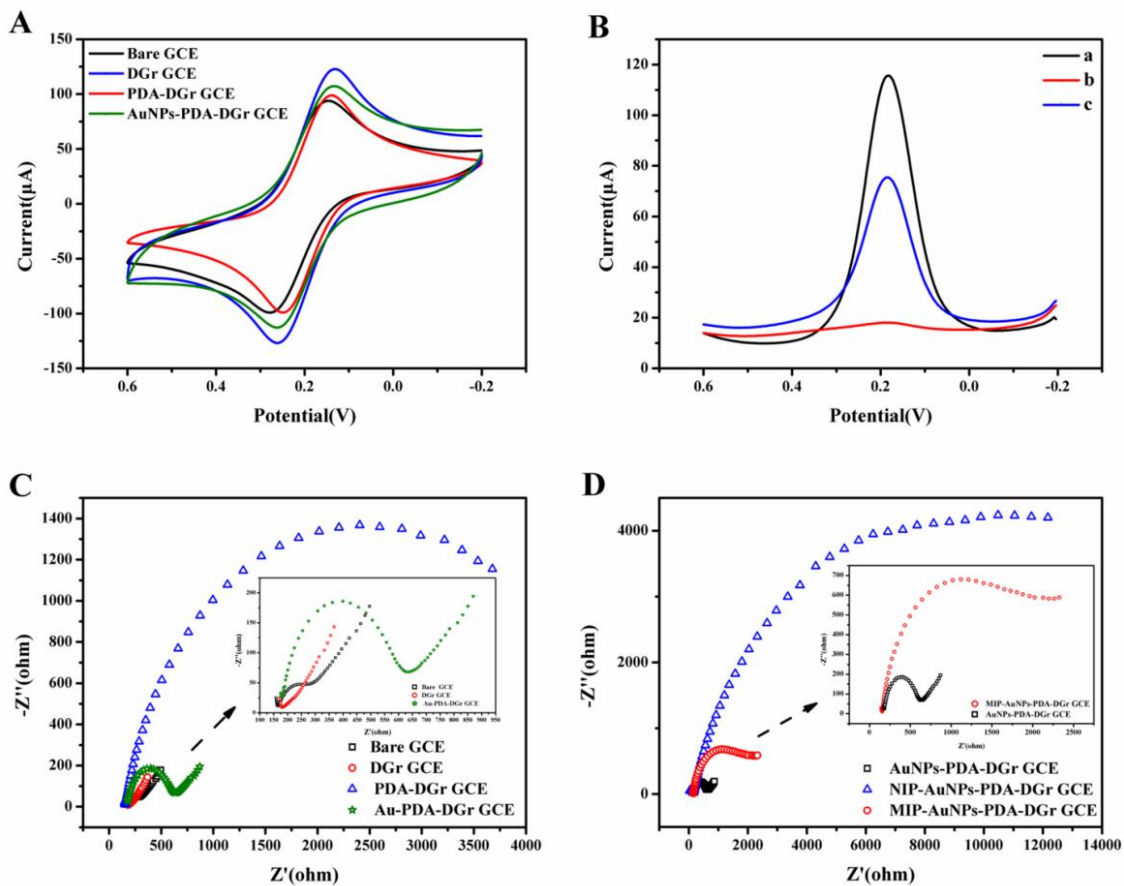

Fig.2 (A) CV for bare GCE, DGr/GCE, PDA-DGr/GCE, and AuNPs-PDA-DGr/GCE in a $5 \mathrm{mM} \mathrm{Fe}(\mathrm{CN}){ }_{6}{ }^{3-/ 4-}$ and $1.0 \mathrm{M} \mathrm{KCl}$ solution (scan rate: $100 \mathrm{mV} \mathrm{s}^{-1}$, scan range: -0.2 to $0.6 \mathrm{~V}$ ); (B) DPV for (a) AuNPs-PDA-DGr/GCE, (b) NIP-AuNPs-PDA-DGr/GCE, and (c) MIP-AuNPs-PDA-DGr /GCE in a $5 \mathrm{mM} \mathrm{Fe}(\mathrm{CN}) 6^{3-/ 4-}$ and $1.0 \mathrm{M} \mathrm{KCl}$ solution (scan rate: $50 \mathrm{mV} \mathrm{s}^{-1}$, scan range: -0.2 to $0.6 \mathrm{~V}$ ). The electrochemical impedance spectra of $5 \mathrm{mM} \mathrm{Fe}(\mathrm{CN})$ $6^{3-/ 4-}$ and $0.1 \mathrm{M} \mathrm{KCl}$ at (C) bare GCE, DGr/GCE, PDA-DGr/GCE, and AuNPs-PDA-DGr/GCE; (D) AuNPs-PDA-DGr/GCE, MIP-AuNPs-PDA-DGr/GCE and NIP-AuNPs-PDA-DGr/GCE. The frequency range is between 1 and $10^{5} \mathrm{~Hz}$ with signal amplitude of $5 \mathrm{mV}$.

EIS is widely used to probe the electron transfer kinetics at the modified electrode. EIS normally includes a semicircular part at higher frequencies and a linear part at lower frequencies, which correspond to the electron-transfer limited process and diffusion process. The semicircle diameter 
represents the charge transfer resistance $\left(\mathrm{R}_{\mathrm{ct}}\right)$ of $\left[\mathrm{Fe}(\mathrm{CN})_{6}\right]^{3-/ 4-}$ redox probe. As shown in Fig.2C, the DGr/GCE shows almost straight lines, which indicates faster electron-transfer kinetics of $\left[\mathrm{Fe}(\mathrm{CN})_{6}\right]^{3-/ 4-}$ compared to bare electrode. However, after the PDA films produced on the modified electrode surface, the $\mathrm{R}_{\mathrm{ct}}(3530 \Omega)$ was enlarged. The AuNPs anchored on the PDA films have higher electrochemical activities so that the $\mathrm{R}_{\mathrm{ct}}(460 \Omega)$ of the AuNPs modified electrode is much smaller than the PDA modified electrode. The impedance of the MIP (2116 $\Omega$ )/NIP (12031 $\Omega)$ modified electrode (Fig.2D) were significantly increased, which indicates p-ATP films have large obstruction effect. Moreover, the impedance of the MIP modified electrode was smaller than that of the NIP modified electrode. This result may be because that the large numbers of the imprinted cavities of $\mathrm{CHO}$ in the MIP films enhanced the diffusion of the redox probe and the electron transfer.

\subsection{Optimization of parameters for MIP-AuNPs-PDA-DGr/GCE.}

The PDA is deprotonated of the phenolic group at high $\mathrm{pH}$, while it is protonated of the amino group at low $\mathrm{pH}$. The effect of $\mathrm{pH}$ of the DA solution on the electropolymerization of PDA (Fig.3A) was investigated by preparing modified electrode in different $\mathrm{pH}$ of DA solution $(4.5,6.5,8.5)$. And we use $\Delta \mathrm{I}$ value (the peak currents of MIP-AuNPs-PDA-DGr/GCE after eluted out the template minus the peak currents of this electrode after absorbed $10^{-15} \mathrm{~mol} \mathrm{~L}{ }^{-1} \mathrm{CHO}$ ) which can represents the adsorption capacity of the MIP films to analyze the effect of $\mathrm{pH}$ of the DA solution. With $\mathrm{pH}$ increasing, the peak currents of the modified electrode after adsorbed CHO molecules decreased. The modified electrode electropolymerized in $\mathrm{pH} 8.5$ of DA solution has larger obstruction effect that the peak currents are the smallest. However, the $\Delta \mathrm{I}$ value of the modified electrode prepared in $\mathrm{pH} 6.5$ of DA solution is larger than the others. So a pH 6.5 of DA solution was selected to electropolymerize the PDA films. 
The molar ratios of template and monomer influence the numbers of the imprinted cavities. As shown in Fig.3B, the high $\Delta \mathrm{I}$ value was obtained when the molar ratios of template and monomer was 1:1. The thickness of the polymer membrane which influences the sensitivity of the MIP sensor by influence the number of the imprinted cavities can be controlled by scanning cycles. We can see that the high $\Delta \mathrm{I}$ value was obtained when scan cycles was 7 (Fig.3C). With the MIP films become more thicker when the scan cycles over 7 cycles, the $\Delta \mathrm{I}$ value was more smaller. This may be because $\mathrm{CHO}$ molecules could not access to the deeply imprinted film. The scan rates for MIP film electropolymerization effect the density of the film. A tight film could decrease the accessibility of template to the imprinted sites. As shown in Fig.3D, the high $\mathrm{I}_{\mathrm{p}}$ value after elution which indicates the recognition capacity was obtained when scan rates are $75 \mathrm{mV} \mathrm{s}^{-1}$. A loose and rough film would produced when a faster scan rate was performed, which would decrease the recognition capacity of MIP film.
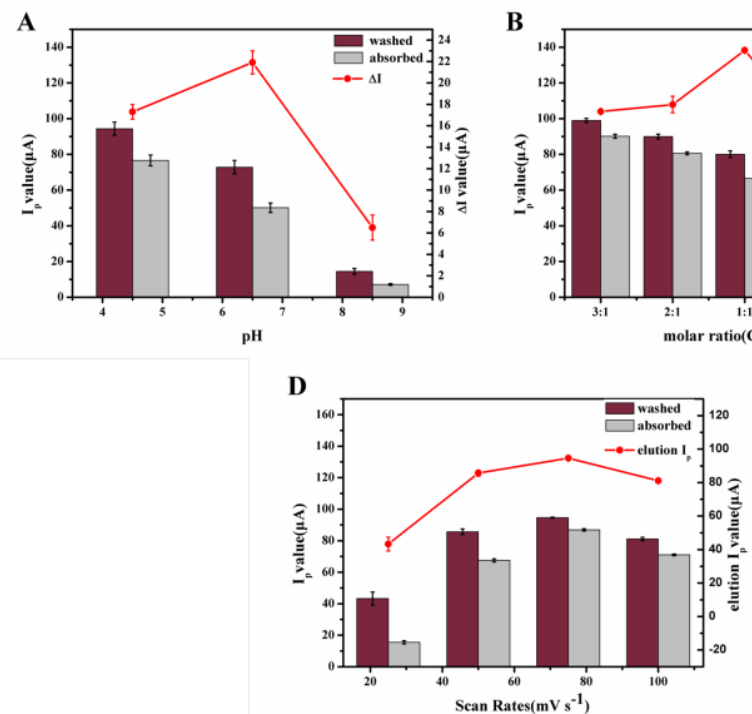
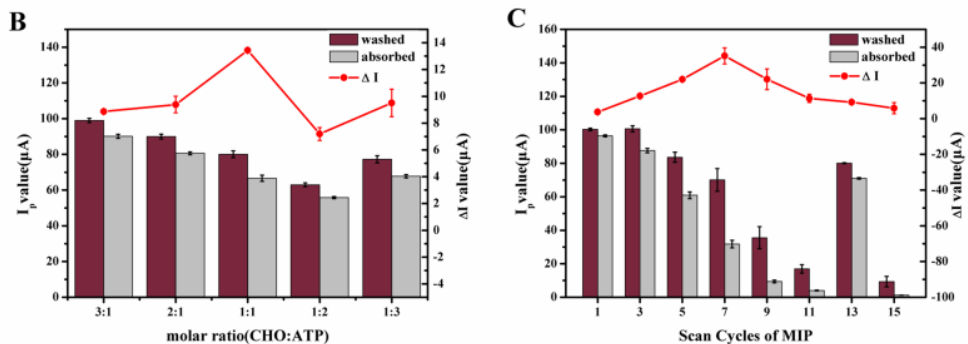

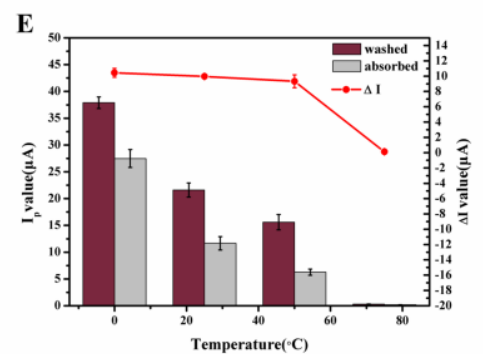

Fig. 3 Effect of (A) pH of electropolymerized DA solution, (B) molar ratio of CHO and ATP, (C) the scan cycles, (D) scan rates, (E) electropolymerized temperature for MIP modified electrode preparation on the peak currents of $10^{-15} \mathrm{~mol} \mathrm{~L}^{-1} \mathrm{CHO}$ recorded on MIP-AuNPs-PDA-DGr/GCE.

The temperature of electropolymerization influences the formation of imprinted sites. The high $\Delta \mathrm{I}$ value was obtained when temperature is $0^{\circ} \mathrm{C}$ (Fig.3E). The reason for this has been postulated on 
the basis of Le Chatelier's principle, which predicts that lower temperatures may increase the number and the quality of the imprinted sites by drive the pre-polymer complex toward complex formation (Spivak, 2005). Above all, we prepared the modified electrode under the optimum conditions. The optimum molar ration between template molecules and monomers was about 1:1. The optimum scanning cycles and the optimum scan rate were about 7 cycles and $75 \mathrm{mV} \mathrm{s}^{-1}$, respectively. The optimum electropolymerized temperature was about $0{ }^{\circ} \mathrm{C}$.

In this work, the MIP modified electrode was immersed in $0.5 \mathrm{~mol} \mathrm{~L}^{-1} \mathrm{HCl}$ ethanol solution and treated at $-0.1 \mathrm{~V}$ constant potential for $40 \mathrm{~s}$ to remove the template. Fig.S5A shows the influences of the potential value on the peak currents of the MIP modified electrode after removal of templates. As shown in Fig.S5A, the optimum potential value was about $-0.1 \mathrm{~V}$. The peak currents increased after eluted for $10 \mathrm{~s}$, then the currents decreased a little and remained stable after 40s shown in Fig.S5B and $\mathrm{C}$. This indicates that the template $\mathrm{CHO}$ molecules were eluted out after $40 \mathrm{~s}$ at the elution condition. The reason why the currents decreased a little after $10 \mathrm{~s}$ may be is that the currents become stable gradually after treated at $-0.1 \mathrm{~V}$ constant potential in $\mathrm{HCl}$ ethanol solution. As shown in Fig.S5D, we also optimized the elution solution. The MIP modified electrode in $0.5 \mathrm{~mol} \mathrm{~L}^{-1} \mathrm{HCl}$ ethanol solution without treated at constant potential could not be cleaned out the templates completely even lasted for 4 min. Similarly, the MIP modified electrode eluted in ethanol solution with treated at constant potential could not even cleaned out with the elution time extending to 60 min. So we choose the $0.5 \mathrm{~mol} \mathrm{~L}{ }^{-1} \mathrm{HCl}$ ethanol solution as elution solution and treated the modified electrode at $-0.1 \mathrm{~V}$ constant potential for $40 \mathrm{~s}$ to remove the template $\mathrm{CHO}$ molecules.

The adsorption time of CHO molecules onto the MIP modified electrode is shown in Fig.S5E and F. The peak currents decreased with the adsorption time increasing, which indicates the amount of 
CHO adsorbed onto the MIP modified electrode increased. The adsorption reached kinetic equilibrium within 5 min.

\subsection{Analytical application of the MIP-AuNPs-PDA-DGr/GCE}

MIP-AuNPs-PDA-DGr/GCE was immersed in different concentration of $5 \mathrm{~mL}$ CHO ethanol solution at room temperature (from $10^{-18} \mathrm{~mol} \mathrm{~L}^{-1}$ to $10^{-13} \mathrm{~mol} \mathrm{~L}^{-1}$ ). The peak currents of CHO on MIP modified electrode was recorded by DPV under the optimized conditions in a solution containing 5 $\mathrm{mM} \mathrm{Fe}(\mathrm{CN})_{6}^{3-/ 4-}$ and $1.0 \mathrm{M} \mathrm{KCl}$ shown in Fig.4. The current responses decreased with the successive addition of $\mathrm{CHO}$, which was because $\mathrm{CHO}$ occupied the imprinted cavities on the MIP and hindered the electron transfer of $\left[\mathrm{Fe}(\mathrm{CN})_{6}\right]^{3-/ 4-}$. Calibration curves in Fig.4B revealed that the peak currents were proportional to the $\log$ of concentrations of $\mathrm{CHO}$ from $10^{-18} \mathrm{~mol} \mathrm{~L}^{-1}$ to $10^{-13} \mathrm{~mol} \mathrm{~L}^{-1}$ with a linear regression equation of $\mathrm{I}(\mu \mathrm{A})=-18.479-3.288 \lg \left[\mathrm{c}\left(\mathrm{mol} \mathrm{L}^{-1}\right)-7.569 \times 10^{-19}\right]\left(\mathrm{R}^{2}=0.997\right)$. The detected limit was $3.3 \times 10^{-19} \mathrm{~mol} \mathrm{\textrm {L } ^ { - 1 }}$. Similarly, the procedure was carried out on NIP-AuNPs-PDA-DGr/GCE for comparison. The fluctuation extent of current of NIP-AuNPs-PDA-DGr/GCE with a linear regression equation of $\mathrm{I}(\mu \mathrm{A})=-1.315-0.136 \lg [\mathrm{c}(\mathrm{mol}$ $\left.\left.\mathrm{L}^{-1}\right)-7.246 \times 10^{-19}\right]\left(\mathrm{R}^{2}=0.997\right)$ influenced by the concentration of $\mathrm{CHO}$ was much weaker compared to MIP-AuNPs-PDA-DGr/GCE. The function of PDA was analyzed and shown in Fig.S6. Besides, we also investigated that a self assembled p-ATP and $\mathrm{CHO}$ monolayer was prepared on the AuNPs-PDA-DGr/GCE surface before electropolymerized MIP films, if the analytical capability of the MIP sensor was improved or not (Fig.S6). It turns out that the best analytical capability was obtained by using MIP-AuNPs-PDA-DGr/GCE to detect trace $\mathrm{CHO}$, which attributed to the synergistic effect of PDA, Au microflowers, DGr and MIP. We compared our work with other published CHO electrochemical sensors and MIECS as well. From table S1 we can obviously see 
that MIP-AuNPs- PDA-DGr/GCE in this work is much more sensitive, compared to previously reported sensor.

A

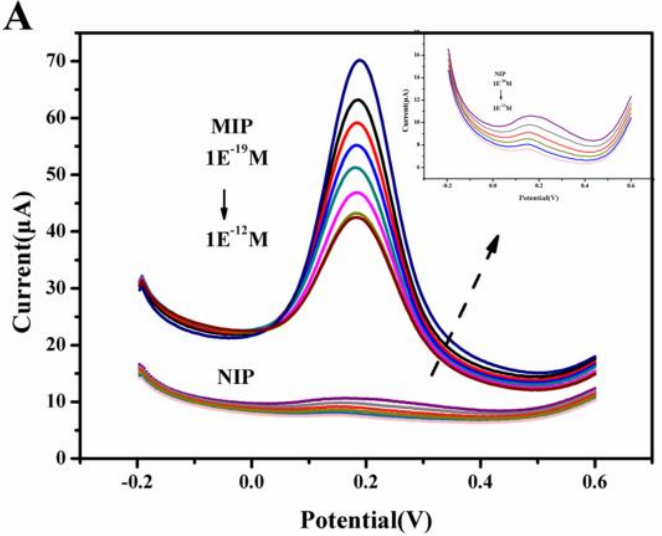

B

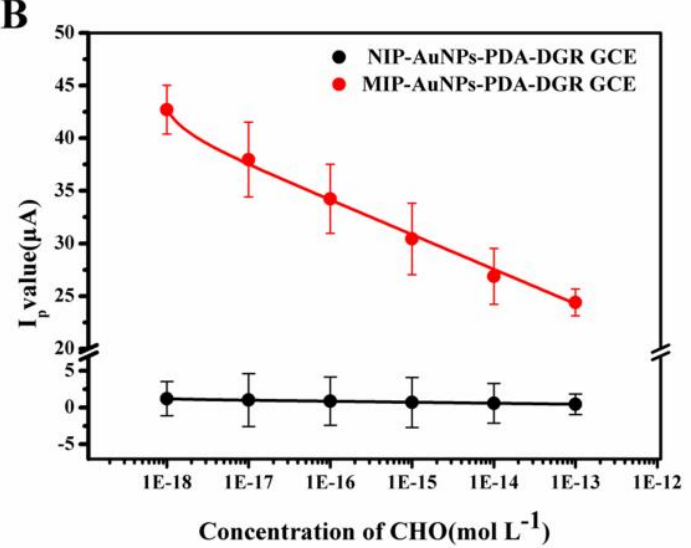

Fig.4 (A)DPV of MIP-AuNPs-PDA-DGr/GCE and NIP-AuNPs-PDA-DGr/GCE incubated in different concentrations of $\mathrm{CHO}$ ethanol solution for $4 \mathrm{~min}$ in a solution containing $5 \mathrm{mM} \mathrm{Fe}(\mathrm{CN}){ }_{6}^{3-4-}$ and $1.0 \mathrm{M} \mathrm{KCl}$ (from $10^{-18}$ to $10^{-13} \mathrm{~mol} \mathrm{~L}^{-1}$ ). Scan rate: $50 \mathrm{mV} \mathrm{s}^{-1}$, scan range: -0.2 to $0.6 \mathrm{~V}$. (B) Relationship of peak values of MIP-AuNPs-PDA-DGr/GCE and NIP-AuNPs-PDA-DGr/GCE with the concentration of CHO (from $10^{-18}$ to $10^{-13}$ mol L ${ }^{-1}$ ).

\subsection{Selectivity, reproducibility and stability of the MIP modified electrode}

The selectivity of the MIP modified electrode was investigated when some possible interfering substances as the control, such as vitamin D3, estradiol, stigmasterol, progesterone, testosterone, estrone, dapamine, ascorbic acid, uric acid. Fig.S7 shows the peak currents of the MIP modified electrode after incubated in $10^{-13} \mathrm{~mol} \mathrm{~L}^{-1}$ vitamin $\mathrm{D} 3$, estradiol, stigmasterol, progesterone, testosterone, estrone, dapamine, ascorbic acid, uric acid and $10^{-18} \mathrm{~mol} \mathrm{~L}-1$ CHO, respectively. Among them vitamin D3 and progesterone have the most similar structures to CHO. The selectivity was evaluated by calculating the $\Delta \mathrm{I}$ ratio $\left(\Delta \mathrm{I}_{\mathrm{o}} / \Delta \mathrm{I}_{\mathrm{i}}\right)$, where $\Delta \mathrm{I}_{\mathrm{o}}$ and $\Delta \mathrm{I}_{\mathrm{i}}$ were $\Delta \mathrm{I}$ of $\mathrm{CHO}$ and $\Delta \mathrm{I}$ of interfering substances. The $\Delta \mathrm{I}$ ratio were only slightly varied from 0.07 to 0.41 . The results indicate that the MIP sensor showed higher recognition selectivity for $\mathrm{CHO}$ than for other interfering substances. This is because the MIP films are expected to create shape selective cavities to template within the polymer structure. 
To study the reproducibility of the measurements, the MIP modified electrode was used to detect $10^{-15} \mathrm{~mol} \mathrm{~L}{ }^{-1} \mathrm{CHO}$ for 7 times eluted and measured. And the peak currents relative standard deviation (RSD) was $2.24 \%$. The reproducibility of MIP sensor was also investigated by detecting CHO on five different GCE with a RSD of 1.9\%. Moreover, the peak current responses of the MIP modified sensor decreased to $97.4 \%$ after storing in elution solution for 40 days at $4^{\circ} \mathrm{C}$. These results indicate that the MIP modified sensor had excellent selectivity, reproducibility and stability.

\subsection{Human serum analysis}

In order to investigate the potential of the MIP sensor for practical application, the MIP sensor was applied to detect $\mathrm{CHO}$ in human serum by the standard addition method. Perchloric acid was added to human serum with a volume ratio of 1:10 ( $\left.\mathrm{V}_{\text {Perchloric acid: }} \mathrm{V}_{\mathrm{CHO}}\right)$ to remove protein. After centrifuged, $1 \mathrm{ml}$ of the above-mentioned serum solution was diluted to $10 \mathrm{ml}$ with ethanol. A series of sample solutions were prepared by adding a certain volume of $\mathrm{CHO}$ to $10 \mathrm{ml}$ human serum samples. The results were listed in Table 1. The results demonstrated that this MIP sensor can be applied to human serum assays.

\section{Table 1}

Application of the MIP modified sensor to detect cholesterol in human serum.

$\mathrm{M}$ is mean and RSD is relative standard deviation.

\begin{tabular}{llllll}
\hline Sample & Added $\left(\mathrm{mol} \mathrm{L}^{-1}\right)$ & Found $\left(\mathrm{mol} \mathrm{L}^{-1}\right)$ & Recovery $(\%)$ & $\mathrm{M}(\%)$ & $\operatorname{RSD}(\%)$ \\
\hline & 0 & - & - & & \\
& $10^{-18}$ & $9.8 \times 10^{-19}$ & 98.0 & & \\
Human & $10^{-17}$ & $1.03 \times 10^{-17}$ & 100.3 & 98.9 & 1.7 \\
Serum & $10^{-15}$ & $1.04 \times 10^{-15}$ & 100.4 & & \\
& $10^{-13}$ & $9.7 \times 10^{-13}$ & 97.0 & & \\
\hline
\end{tabular}




\section{Conclusions}

In this work, we explore a novel and sensitive sensor for trace $\mathrm{CHO}$ determination based on electropolymerized molecular imprinting at the surface of DGr and versatile Au microflowers, which was wrapped by bioinspired PDA film. Gr was reduced by DA in weak alkaline phosphate buffer solution $(\mathrm{pH}=8.5)$ to prepare DGr, which was the substrate of the MIP sensor. Au microflowers produced a high ratio of imprinted sites and acted as crosslinkers to form polymeric networks through $\mathrm{Au}-\mathrm{S}$ between Au and the functional monomer ATP. PDA films have metal binding ability to stabilize AuNPs and can form intermolecular forces between ATP and PDA. The lowest detectable concentration of $\mathrm{CHO}$ was $3.3 \times 10^{-19} \mathrm{~mol} \mathrm{~L}^{-1}$ and the linear detection range was from $10^{-18}$ to $10^{-13}$ mol L ${ }^{-1}$. Except the advantages of low cost, wide linear range, low detection limit, good selectivity, and good stability, this MIP sensor was also successfully applied to detect CHO in human serum with high recovery. From the above conclusion, PDA films could act as a novel bioinspired multifunctional modified materials in MIECS and help capture more template molecules. These results suggest that this method can also be developed for other molecules selective recognition and sensitive detection. 


\section{Acknowledgement}

The authors would like to thank the National Science Foundation of China (No.21271127, 21671132) and partly supported by Shanghai Key laboratory of High Temperature Superconductors (No.14DZ2260700) for financial supports.

\section{Reference}

Agnihotri, N., Chowdhury, A.D., De, A., 2015. Biosens. Bioelectron. 63, 212-217.

Ahmadraji, T., Killard, A. J., 2013. Anal. Methods. 5, 3612-3625.

Ali, A., Mohammad, R., Mostafa, N., 2010. Electrochim. Acta. 55, 1503-1508.

Altintas, Z., Gittens, M., Guerreiro, A., Thompson, K.-A., Walker, J., Piletsky, S., 2015. Anal. Chem. 87, 68016807.

Amiri, M., Amali, E., Nematollahzadeh, A., 2015. Sens. Actuators B: Chem. 216, 551-557.

Batra, N., Tomar, M., Gupta, V., 2015. Biosens. Bioelectron. 67, 263-271.

Cao, J., Mei, S., Jia, H., Ott, A., Ballauff, M., 2015. Langmuir. 31, 9483-9491.

Chou, L., Liu, C.C., 2005. Sens. Actuators B: Chem. 110, 204-208.

Dong, J., Guo, H., Yang, R., Li, H.,Wang, S., Zhang, J., Zhou, W., Chen, W., 2012. Clin. Chim. Acta. 413, 10711076.

García-Llatas, G., Vidal, C., Cilla, A., Barberá, R., Lagarda, M.J., 2012. Eur. J. Lipid Sci. Technol. 114, 520-526.

Giri, A.K., Sinhamahapatra, A., Prakash, S., Chaudhari J., Shahi V.K., Panda, A.B., 2013. J. Mater. Chem. A. 1, 814 $-822$.

Hojo, K., Hakamata, H., Kusu, F., 2011. J. Chromatogr. B. 879, 751-755.

Huan, J., Liu, Q., Fei, A.R., Qian, J., Dong, X.Y., Qiu, B.J., Mao, H.P., Wang, K., 2015. Biosens. Bioelectron. 73, 221-227.

Ji, J., Zhou, Z., Zhao, X., Sun, J., Sun, X., 2015. Biosens. Bioelectron. 66, 590-595.

LaVoie, M.J., Ostaszewski, B.L., Weihofen, A., Scholssmacher, M.G., Selkoe, D.J., 2005. Nat. Med. 11, 1214-1221.

Lee, H., Dellatore, S.M., Miller, W.M., Messersmith, P.B., 2007. Science. 318, 426-430.

Liu, Q., Wang, N.Y., Caro, J., Huang, A.S., 2013. J. Am. Chem. Soc. 135, 17679-17682.

Luo, J., Jiang, S., Liu, X., 2013. J. Phys. Chem. C 117, 18448-18456.

Malitesta, C., Mazzotta, E., Picca, R.A., Poma, A., Chianella, I., Piletsky, S.A., 2012. Anal. Bioanal. Chem. 402, 1827-1846.

Nantaphol , S., Chailapakul, O., Siangproh, W., 2015. Biosens. Bioelectron. 207,193-198.

Pierpont, C.G., Lange, C.W., 1994. Prog. Inorg. Chem. 41, 331-442.

Piletsky, S.A., Piletskaya, E.V., Sergeyeva, T.A., Panasyuk, T.L., Elskaya, A.V., 1999. Sens. Actuators B: Chem. 60, 216-220.

Ram, M.K., Bertoncello, P., Ding, H., Paddeu, S., Nicolini, C., 2001. Biosens. Bioelectron. 16, 849-856.

Riskin, M., Tel-Vered, R., Bourenko, T., Granot, E., 2008. JACS. 130, 9726-9733.

Ruecha, N., Siangproh, W., Chailapakul, O., 2011. Talanta, 15, 1323-1328.

Safavi, A., Farjami, F., 2011. Biosens. Bioelectron. 26, 2547-2552.

Sion, B., Grizard, G., Boucher, D., 2001. J. Chromatogr. A. 935, 259-265.

Spivak, D.A., 2005. Adv. Drug Delivery Rev. 57,1779-1794. 
Suryanarayanan V., Wu C.T., Ho K.C., 2010. Electroanal. 22, 1795-1811.

Teng, Y., Fan, L.M., Dai, Y.L., Zhong, M., Lu, X.J., Kan, X.W., 2015. Biosens. Bioelectron. 71, 137-142.

Tong, Y., Li, H., Guan, H., Zhao, J., Majeed, S., Anjum, S., Liang, F., Xu, G., 2013. Biosens. Bioelectron. 47, 553-558.

Tripathy, N., Ahmad, R., Kim, E. Y., Khanga, G., Hahn, Y.B., 2014. Communication. 4, 45049-46053.

Umar, R., Ahmad, S.W., Hwang, S.H., Kim, A., Al-Hajry, Y.B., Hahn, 2014. Electrochim. Acta. 135, 396-403.

Web of Science ${ }^{\mathrm{TM}}$, Thomson Reuters. 〈 http://apps.webofknowledge.com〉 (accessed 20.01.15).

Xie, C.G., Li, H.F., Li, S.Q., Wu, J., Zhang, Z.P., 2010. Anal. Chem. 82, 241-249.

Xu, S.Y., Han, X.Z., 2004. Biosens. Bioelectron. 19, 1117-1120.

Zan, G., Wu, Q., 2016. Adv. Mater. 28, 2099-2147.

Zhang, M., Zhang, X., He, X., Chen, L., Zhang, Y., 2014. Nanoscale. 4, 3141-3147.

Zhou, W.H., Lu, C.H., Guo, X.C., Chen, F.R., Yang, H.H., Wang, X.R., 2010. J.Mater.Chem. 20, 880-883. 